\title{
Incisional and Ventral Hernia Repair
}

\author{
Youn-Baik Choi, M.D., Ph.D. ${ }^{1}$, In-Seob Lee, M.D., Ph.D. ${ }^{2}$ \\ Department of Surgery, 'Chung Hospital, Seoul, and ${ }^{2}$ Ulsan University College of Medicine and Asan Medical Center, Seoul, Korea
}

Keywords: Incisional hernia, Ventral hernia, Laparoscopic hernia repair

This is an Open Access article distributed under the terms of the Creative Commons Attribution Non-Commercial License (http:/ creativecommons.org/licenses/by-nc/4.0/) which permits unrestricted non-commercial use, distribution, and reproduction in any medium, provided the original work is properly cited.

\begin{abstract}
Incisional or ventral hernia is a very common multifactorial disease that requires surgery to prevent complications, including pain, discomfort, bowel obstruction, incarceration, and strangulation. To perform herniorrhaphy, it is essential to understand the pathogenesis of hernia, the anatomy and physiology of the abdominal wall, and surgical techniques. Several repair methods are available, including open suture repair, open mesh repair, the component separation technique, and tissue expansion assisted closure. Currently, laparoscopic incisional or ventral hernia repair is commonly used with the major advantage being the lower recurrence and all defects can be addressed at the time of surgery as well as reduced postoperative pain and length of hospital stay. On the other hand, to do it properly, a full understanding and appropriate selection of mesh and management of probable complications, such as seroma, bowel injury, enteric fistula, and recurrence, is essential. Therefore, the surgeon and the techniques used are of paramount importance in the repair of incisional ventral hernias.
\end{abstract}

Received April 25, 2017

Revised 1st November 8, 2017 2nd November 10, 2017

Accepted November 14, 2017

Corresponding author

Youn-Baik Choi

Department of Surgery, Chung

Hospital, 550 Olympic-ro,

Gangdong-gu, Seoul 05391, Korea

Tel: $+82-2-2202-3114$

Fax: +82-2-474-9027

E-mail: younbchoi@gmail.com
Copyright @ 2018 The Journal of Minimally Invasive Surgery. All rights reserved.

\section{INTRODUCTION}

Incisional hernia is defined as an abdominal wall defect at the site of abdominal wall closure and more than $10 \%$ of patients, who undergo laparotomy, experience the hernia., It is estimated the number of incisional hernia repair cases would reach 90,000 in USA, 41,000 in Germany ${ }^{3}$ and 2,150 in Korea per year. Approximately $50 \%$ of incisional hernia develop or present within the 2 years following surgery, and $74 \%$ occur within 3 years. ${ }^{4,5}$ Ventral hernia is a bulge of tissues through an opening of weakness within abdominal wall muscles without surgery.

There is an ongoing discussion concerning which factor contributes to such situation; is there a 'hernia disease' or simply the coincidence of several conditions or even a physiological reaction to tensile forces stretching the abdominal wall. Predisposing factors are obesity, diabetes, emergency surgery, postoperative wound dehiscence or infection, smoking, immunosuppression, prostatism, and collagen disorders such as abdominal aortic aneurysm and Ehlers-Danlos Syndrome.,7 Rosch and colleagues favor the inability to form physiological collagen as an essential cause for the first herniation as well as for the recurrence of such hernias. ${ }^{8}$ He found decreased collagen deposition and the reduced collagen I - collagen III ratio in hernia scars. Degradation of connective tissue caused by an imbalance between proteases and their inhibitors has also been postulated. The techniques for abdominal closure are important, but it was debated and "surgeon-dependent" variables. The incidence was as great as 3 times in absorbable suture materials and there was no significant difference between continuous and interrupted closure.

Although the incisional hernia may stay silent and asymptomatic for years, it may enlarge over time and can give rise to complications including pain, discomfort, bowel obstruction, 
incarceration, and strangulation. Also it may adversely affect an individual's quality of life. About $17 \%$ will lead to incarceration or strangulation with $0.3 \%$ mortality, so early surgical intervention is important once the incisional hernia has been diagnosed.

Repairing incisional hernia requires many problems to be overcome: a multi-layered wall structure of different tissue properties in constant motion has to be sutured, positive abdominal pressure has to be dealt with, and tissues with impaired healing have to be joined. ${ }^{9}$ Also we should explain to the patient of the risks of repairing an incisional hernia including seroma formation, wound infection, mesh infection, injury to the intra-abdominal structures, and enterocutaneous fistula which may result in prolonged morbidity and require re-operation, and recurrence.

The goals of incisional hernia repair are the prevention of visceral eventration, incorporation of the abdominal wall in the repair, provision of dynamic muscular support, and restoration of abdominal wall continuity in a tension-free manner.

\section{BODY TEXT}

\section{Open suture repair}

In the past, many of these hernias have been treated with abdominal trusses and several operations. Before 1963, most incisional hernias were repaired by direct suture techniques including simple fascial closure, modified Mayo technique with overlap of fascial edges, use of internal retention sutures, the Maingot 'Keel' procedure using relaxing incisions in the lateral aspect of the anterior rectus sheath, the 'Nuttall' procedure involving transposition of rectus abdominis and its enveloping fascia, use of layered steel wire and others.

The common complications after open suture repair are wound-related problems including infection, hematoma, stitch sinus, and flap necrosis. These occurred in 10 44\%. ${ }^{10}$ In spite of these poor results, suture techniques have been used continuously.

Suture repair for incisional hernia is archaic, so surgeons should consider abdominal incisions that have a lower incidence of incisional hernia than mid-line incisions (i.e. paramedian and transverse incision). The rate of recurrence following these repairs is reported between $11 \sim 52 \%$. ${ }^{11,12}$ These high recurrence rates prompted recommendations of 'a cautious attitude to surgical treatment of incisional hernia' in the mid-1980s, and led to the widespread acceptance of mesh repair. $^{13}$

In pre-menopausal women, it is required to allow subsequent pregnancy with elasticity and expansion of the abdominal wall. So it may be better to avoid mesh and use sutured repair such as 'shoelace' technique in this situation.

\section{Open mesh repair}

A wide spectrum of surgical techniques has been developed and recommended, ranging from suture techniques to the use of various types of prosthetic mesh. Usher introduced knitted monofilament polypropylene (Marlex) mesh into clinical practice in 1963, which is still the most widely used prosthetic material. In 1972, Gore et al developed polytetrafluoroethylene (PTFE) and it is gaining popularity because of its apparent reduced tissue reaction. Various studies from mesh repair of incisional hernia have been reported. ${ }^{14}$

The open mesh repair technique involves placement of a large prosthetic mesh in a retro-muscular, extraperitoneal location. The mesh overlaps the margins of the incisional hernia by several centimeters and is secured by multiple, interrupted, transabdominal sutures placed along the edge of the prosthetic mesh. The choice of open mesh technique is onlay, sublay, and inlay method (Fig. 1). Notaras implanted Mersilene mesh deep to the rectus muscle (sublay) with its edges at least 2.5 $\mathrm{cm}$ beyond the edges of the defect and administered antibiot-
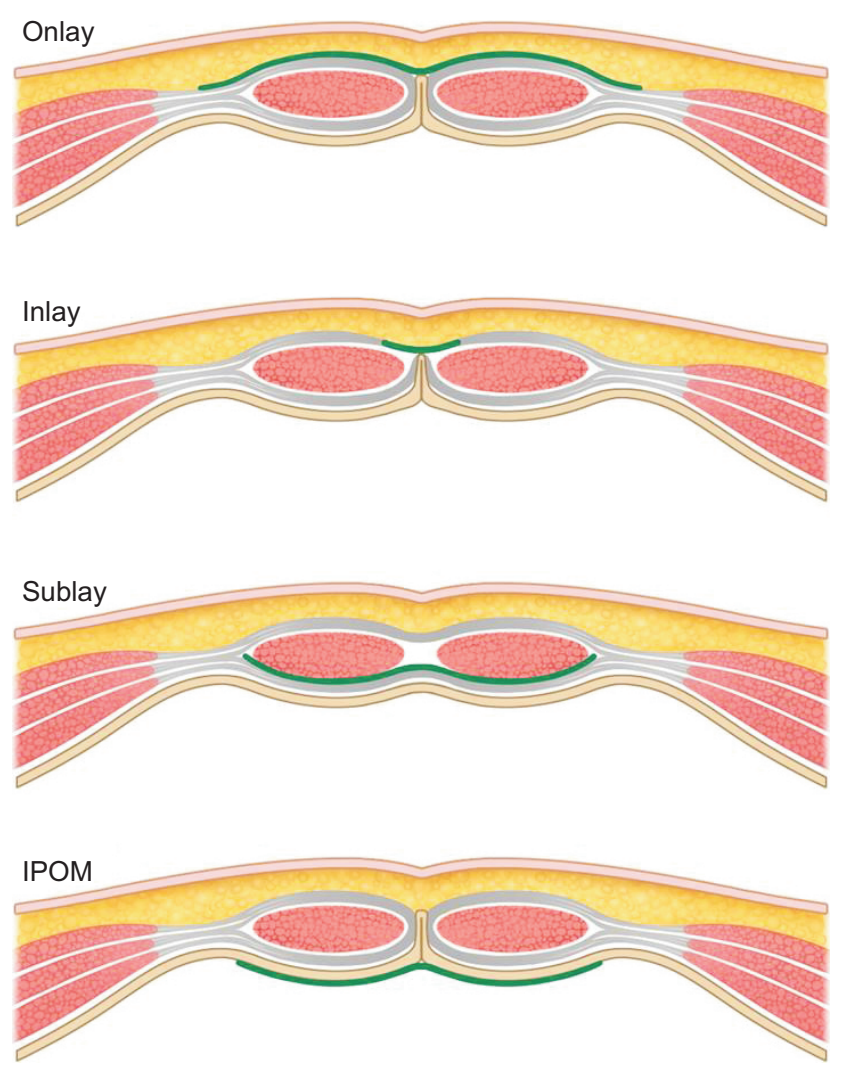

Fig. 1. Several techniques of mesh repair for incisional or ventral hernia according to the location of mesh placement. 
ics prophylactically with the use of closed suction drainage postoperatively. The recurrence rates are markedly less than that for suture repair. It varies 0 and $10 \%$ with a follow-up period of at least 12 months. Development of mesh enabled several methods including the prefascial subcutaneous or onlay method and the inlay technique where the fascial edges are not approximated and the mesh lies in contact with the underlying viscera. A cochrane review concluded that there was insufficient evidence to recommend which method gave the best results. ${ }^{15}$

\section{1) The inlaytechnique}

It involves excision of the hernia sac and identification of healthy fascial margin. For bridging the fascial defect, polypropylene mesh anchors to all adjacent tissues and has the propensity for inducing extensive adhesions to viscera if placed in a position where they become adjacent to bowel in this technique. Erosion of the mesh may then occur into the intestine, so it is recommended to use an expensive doublelayered mesh with an inner layer with non-adhesive coating. As these meshes do not restore the anatomy and physiology of the anterior abdominal wall, activities that increase intraabdominal pressure impact significant tension on the meshfascial interface, which is the weakest point of the repair. The reherniation rate of this technique tends to be higher than those of the underlay and onlay technique. ${ }^{16,17}$

\section{2) The onlay technique}

It consists of relaxing incisions in the anterior rectus sheath with primary approximation of the linea alba and medial turnover of the anterior rectus sheath followed by mesh placement. The disadvantages are that it requires wide undermining of tissue, which may predispose the patient to wound-related complications, and that less pressure is required for disrupting the mesh from the anterior abdominal wall than that of other repair procedures.

\section{3) The underlay technique}

In retrorectal underlay method, the mesh is placed between the posterior rectus sheath and transverse fascia, beneath the rectus muscle. In the preperitoneal underlay technique, the mesh is placed between the transverse fascia and peritoneum. This is a complex operation and is only applicable to mid-line hernias and, in the lower one-third of this region, the mesh is only protected from bowel by tenuous peritoneum. Recurrence rates of less than $10 \%$ have been reported. ${ }^{18}$

\section{The component separation technique}

Since its original description by Ramirez et al, in $1990,{ }^{19}$ this technique has been increasingly used as a tensionless closure of large, full thickness anterior abdominal wall defects with autologous tissue. This classic components separation technique involves the following:

A. The longitudinal release of the medial edge of the external oblique aponeurosis (approximately 1.5 2 $\mathrm{cm}$ lateral to the linea semilunaris to avoid injury to intercostals nerves), followed by blunt separation of the external oblique muscle from the internal oblique muscle in an avascular plane out to the anterior axillary line.

B. Separation of the rectus abdominis muscles from the underlying posterior rectus sheath.

It is a natural method of fascia-fascia closure without the complication of an artificial implant caused by creation of a linea alba, which provides a midline anchor. ${ }^{20}$ This repair allows for advancement of the rectus abdominis muscle up to $10 \mathrm{~cm}$ per side, fascilitating closure of large gaps of the abdominal wall. An obvious prerequisite for this technique is the presence of undamaged rectus muscles. However, this operation will allow the tensionless approximation of the rectus muscle in large (as large as $35 \mathrm{~cm}$ in transverse diameter) and recurrent hernias precluding the problem of abdominal compartment syndrome. ${ }^{21}$ Many surgeons recommend the additional application of synthetic mesh in an onlay position to supplement the attenuated layers of the anterior abdominal wall. $^{22}$

\section{Loss of domain}

Some abdominal wall defects are so large that contents are irreducible owing to an abdominal wall that is chronically injured and reduced. This is referred to as "loss of domain". So returning these contents will require significant physiological (mainly respiratory) adaptation if the volume exceeds more than $15 \sim 20 \%$ of this compartment. ${ }^{23}$ It is essential to require careful patient selection, and surgical team involving plastic surgeons, anesthesiologists, ICU care, and preoperative considerable weight loss. The component separation technique is a valuable option in the repair of loss of domain.

\section{Tissue expansion-assisted closure}

In order to expand tissue prior to incisional hernia repair, tissue expanders were placed in the subcutaneous or submuscular space for months. It is particularly useful in defects of the abdominal wall occurring after major trauma, tumor ablation or congenital abnormalities. ${ }^{24}$ 


\section{Other repair techniques}

Include tissue bank cadaveric grafts, autologous myocutaneous flaps, and healing by secondary or tertiary intention.

\section{Laparoscopic repair}

Since reporting the first case of laparoscopic incisional hernia repair with the use of synthetic mesh in $1993^{7}$ based on the open technique popularized by Rives-Stoppa operation, ${ }^{25-27}$ it has evolved worldwide in recent years and carries many advantages such as reduced postoperative pain, length of hospital stay, and recurrences in comparison with conventional open approach. ${ }^{28}$

Indications of laparoscopic repair are as follows: symptoms such as pain, abdominal enlargement, and risk of incarceration, especially hernia sacs with a small neck that contain bowel. Dumainian and Denham stated that transverse size of $10 \mathrm{~cm}$ is the upper limit for this approach, but the best candidates are middle-sized hernias between $10 \sim 15 \mathrm{~cm}$ in transverse diameter in which the contents can be easily reduced. ${ }^{29}$ Those over $15 \mathrm{~cm}$ in transverse dimension usually require an open supplementary component seperation operation. Contraindications include inability to create a working space, acute or emergency procedure (ie, bowel obstruction), infection of skin or surrounding structures overlying the repair (all infection must be treated and cured before the procedure), ascites with Child class ' $C$ ' cirrhosis, those with loss of domain (because the contents of the hernia sac cannot be reduced), and open wounds (insufflation is impossible) where additional gastrointestinal surgery is required. Occasionally due to unusual dense adhesions, patients who have had a previous incisional hernia repair with mesh placement are contraindicated. Though obese patients should be consulted regarding the increased risk for hernia recurrence, obesity is not a contraindication. For them, a bariatric evaluation is recommended with encouraging them to lose weight preoperatively if possible.

The locations of incisional hernia are mid-line incision ( 77\%), lateral incision ( $17 \%)$ and iliac incision $(\sim 6 \%) .{ }^{30}$ Upper midline incision has a high incidence of hernia formation than other type of incision do. It is explained that the configuration of the collagen bundles of the abdominal wall are oriented transversely, so a transverse suture line is mechanically more stable, as it encircles the fibers rather than splitting them. ${ }^{31}$

This technique requires placement of three or more port sites as far away as possible from the defect to allow adhesiolysis and reduction of the contents of the hernia sac, visualization of the hernia defect, and intraperitoneal placement of mesh that overlaps the defect (by at least $3 \mathrm{~cm}$ ) in all directions. It is advisable to locate trocars perpendicular to the $\mathrm{ab}^{-}$ dominal wall and not to close to ASIS (Anterior Superior Iliac Spine) because excessive adipose tissue over the ASIS interfere with instruments through trocars. Many surgeons prefer the distance from the defect to two working trocars to about $10 \mathrm{~cm}$ and the degree from the defect to working ports to $45^{\circ} \sim 60^{\circ}$ (Fig. 2). This allows a large surface area laterally for ingrowth of connective tissue, leading to permanent fixation of the prosthesis within the abdominal wall.

The major advantage may underlie the lower recurrence of the laparoscopic technique as all defects can be addressed at the time of surgery. Clinical examination is often misleading because of multiple small defects (swiss-cheese defects) may be associated with a previous incision. Comparing Banerjea ${ }^{32}$ and our series, ${ }^{33}$ the mean number of defects per patient noted was (2.7:3.2) compared to (1.2:1.3) defects detected at clinical examination preoperatively. If we cannot find the definite defects, it is necessary to switch the scope position, and consider placing a fourth $5 \mathrm{~mm}$ trocar opposite to the placement of other trocars. ${ }^{34}$

Iatrogenic enterotomy is a serious problem during laparoscopic repair with an incidence from 0 to $14 \%$. The worst surgical outcome with enterotomy is failure to recognize them in postoperative period (mortality $40 \%$, morbidity $100 \%$ ). It can be avoided by two-handed technique, using a meticulous technique and sharp dissection to avoid thermal injury, keeping intraabdominal pressure high during dissection and low during closing, looking at bowel at the end of the procedure, and it is essential for the surgeon to be patient during entire procedure. The most common location of injury was the small bowel (55.8\%) followed by the large intestine (38.6\%) and, less commonly, the stomach (3.9\%). Enterotomy during

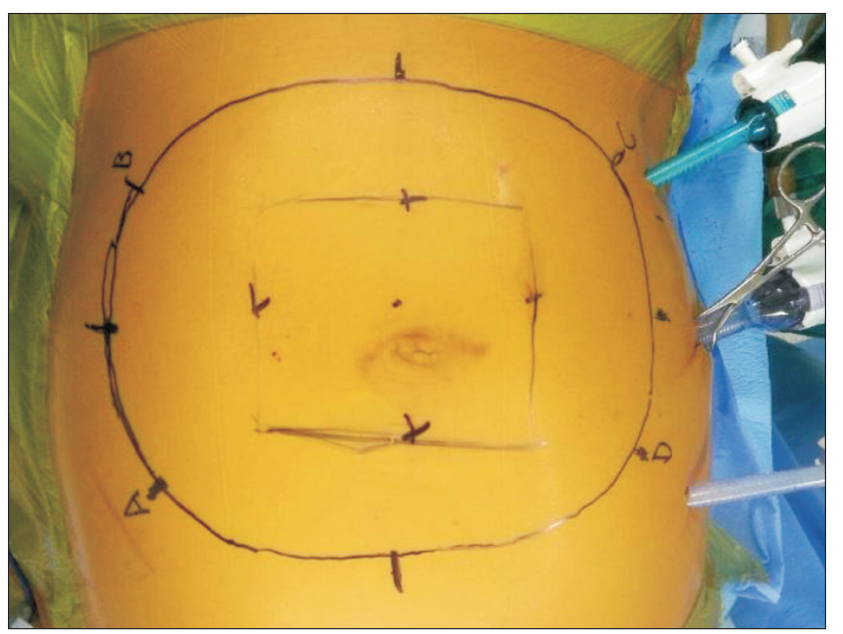

Fig. 2. Placement of ports for the laparoscopic repair of incisional hernia. 3 trocars are usually used. A $12 \mathrm{~mm}$ camera trocar is located in the middle of trocars and two $5 \mathrm{~mm}$ trocars are placed in both sides. 
this technique has been reported between 1 6\% and usually occurs during adhesiolysis. ${ }^{36}$ When iatrogenic enterotomy occurred, the method chosen to repair was generally determined by the extent of the injury and the skill level of the surgeon. If significant contamination does not exist, the repair was completed with laparoscopically placed mesh. On the other hand, if significant contamination does exist, the repair can either be performed by the open tissue repair method at the initial operation or laparoscopically with the placement of mesh after delaying within one week. ${ }^{37}$ LeBlanc and Heniford stated that it may be permissible to repair the hernia with prosthesis even in the presence of a colonic injury if an antimicrobialimpregnated prosthesis is used, but more study in this area is warranted. ${ }^{21,38}$

There are 3 options for sizing of the mesh; intracorporeal with pneumoperitoneum, extracorporeal with pneumoperitoneum and extracorporeal desufflated method.

The use of mesh has increased from 34.2\% in 1987 to 65.5\% in 1999. Four main types of prosthetic mesh have been used: polypropylene (Prolene; Ethicon, Somerville, New Jersey), ePTFE (Dual mesh; Gore-Tex; Gore Medical, Flagstaff, Arizona), Composite polypropylene+collagen (Parietene; Sofrad- im, Trevoux, France). Because of creating adhesions and fistulization with bowel loops, polypropylene has been replaced by Proceed (Ethicon), which is composed of polypropylene covered with oxidized regenerated cellulose (ORC)..$^{39}$ A newer mesh composed of polypropylene covered by a layer of polyglecaprone-25 on both sides (Physiomesh; Ethicon) has been added recently. The new Gore-Tex Dual Mesh Biomaterial, ePTFE, is a patch with two different properties: one side with pore size of $<3 \mu \mathrm{m}$, resulting in minimal tissue attachment, and the other side with a $17-\mu \mathrm{m}$ pore size, which allows host tissue incorporation (Table 1). Biological meshes are mainly used to reconstruct the abdominal wall in an infected field, but they are of limited use in incisional hernia repair because of cost.

Assisted by an Endo-suture passer, the 4 8 transfascial sutures are used to fix the mesh to the anterior abdminal wall, ${ }^{39}$ avoiding postoperative migration of mesh and holding the mesh close to the abdominal wall for excellent tissue incorporation. The mesh is further secured with 5-mm titanium tacs, applied with a Protack (I-clip, Covidien, Mansfield, Massachusetts); with absorbable tacs, applied with the Absorbatack device (Covidien); with the SorbaFix (Bard Devol); using

Table 1. Meshes used intraperitoneally for the repair of ventral and incisional hernias

\begin{tabular}{lll}
\hline \multicolumn{1}{c}{ Group/Mesh } & \multicolumn{1}{c}{ Material } & \multicolumn{1}{c}{ Company } \\
\hline PTEF & ePTFE & Bard Davol, Inc., Warwick, Rl \\
Dulex & ePTFE & W. L. Gore, Newark, DE \\
Mycromesh & ePTFE & W. L. Gore \\
Dual Mesh & & \\
Composite mesh with absorbable coated barrier & PP with ORC layer & Ethicon, Somerville, NJ \\
Proceed & PP with collagen coated & Covidien, Mansfield, MA \\
Parietene & Polyester with collagen coated & Covidien \\
Parietex composite & Polyester with collagen film & Covidien \\
Symbotex & Porcine dermal collagen implant & Covidien \\
Permacol & PP with polyglecaprone 25 & Ethicon \\
Physiomesh & PP with omega 3 fatty acid coating & Atrium Medical, Hudson, NH \\
C-Qur & PP with hydrogel safety coating & Bard Davol, Inc. \\
Sepramesh IP Composite & & \\
Composite mesh with permanent coated barrier & PP/ePTFE & Bard Davol, Inc. \\
Composix & PP/ePTFE & Bard Davol, Inc. \\
Ventrio & PP/ePTFE & Cousin Biotech, Wervicq-Sud, France \\
Intramesh T1 & Polyester mesh with silicone layer & Cousin Biotech \\
Intramesh W3 & PRC & \\
\hline
\end{tabular}

ePTFE $=$ expanded polytetrafluoroethylene; $\mathrm{PP}=$ polypropylene; $\mathrm{ORC}=$ oxidized regenerated cellulose. 
titanium clips (EMS; Ethicon), or fibrin sealant. A recently introduced fixation device, the Secure Strap (Ethicon) uses absorbable straps to fix mesh, with promising results. ${ }^{40}$ Various types of fixation method have been devised; double crown technique, single crown tacking+absorbable sutures, and single crown tacking+non-absorbable sutures fixation. ${ }^{41}$ After tying the knot, pull the transfascial sutures from the skin outwards to release any tension. Disadvantages of transfascial fixation suture are poorer cosmetic result and pain during the early postoperative period. Metal tacks, usually in two rings to form a 'double crown' or a combination of transfascial sutures and tacks may also be used for fixation of the mesh to the abdominal wall with full thickness. The 4-mm long Tack (Protack) penetrates only $2 \mathrm{~mm}$ into the abdominal wall after allowing $1 \mathrm{~mm}$ for thickness of the mesh and another $1 \mathrm{~mm}$ for the tack profile. In obese patient having a substantial amount of preperitoneal fat, the $2 \mathrm{~mm}$ penetration of the tack will not reach the muscle or fascia in most cases. Also the tensile strength of the transfascial suture is 2.5 times greater than that of the tack. It is the reason why the transfascial suture is essential for the laparoscopic incisional hernia repair in obese patient especially.

Seroma was a prominent sequelae postoperatively. It is usually asymptomatic; however 30 35\% patients experience symptoms, such as pain, pressure, and erythema. Non-reducible hernia, multiple incisions, recurrent hernia, and suture placement through the hernia sac during the repair are risk factors. But there was no evidence that specific mesh type was associated with seroma formation. Since most seromas resolve spontaneously without intervention, expectancy is reasonable. Aspiration is justified in large symptomatic cases. Other measures for prevention of seroma are cauterizing the hernia sac, primary fascial closure, and compression dressing for one week after surgery. ${ }^{33,42}$

Abdominal bulging is another problem, representing $1.6 \sim 17.4 \%$ after the laparoscopic repair of large incisional hernia. It may be managed expectantly, if it is asymptomatic. Orenstein et al recommended routine closure of the hernia defect (the "shoelacing" technique) for eliminating postoperative seroma and a reduced abdominal bulging. ${ }^{43}$

The recurrence rate is reported 2.1 18\% in laparoscopic repair and 11 52\% in open repair. ${ }^{32,44}$ Morbid obesity, previous failed open repair, the size of the defect and number of defect, and postoperative complications are associated with an increased risk of recurrence. The larger the defect and the higher the number of defects, the larger is the size of mesh required and the greater is the degree of undermining required. This explains the greater incidence of seroma, hematoma, and wound infections.

Although mesh repair is tension-free, it is a foreign mate- rial and susceptible to infection, sinus formation, enteric fistularization and possible extrusion in 16 18\%. Before surgery, any known risk factor for surgical-site infection should be treated. To reduce the risk of perioperative infection, application of surgical drape such as Iovan ${ }^{\circledR}$ (3M, Healthcare, Neuss, Germany) and aseptic handling of the mesh are needed. In obese patients, high recurrence rate is due to increased intraabdominal pressure. BMI has been shown to directly correlate with increasing intra-abdominal pressure. ${ }^{45}$

\section{CONCLUSION}

On the strength of the advances in the understanding of the anatomy and physiology of the abdominal wall, the choice of suture materials, the knowledge of closure techniques, and the development of prosthetic materials the management of incisional hernia continues to evolve. But it is considered as a challenging procedure, especially in recurrent hernia, in which the chances of recurrence increase with each surgical technique. Successful repair relies on knowledge of the dynamics of the abdominal wall, thorough technical execution, appropriate selection of synthetic or bioprosthetic material, and constitution of surgical team. Though laparoscopic repair has been demonstrated to be safe and a more resilient repair than open repair, open mesh repair remains a suitable alternative.

We should keep in mind that the surgeon and the techniques used are of paramount importance in the repair of incisional ventral hernia.

\section{REFERENCES}

1) Bloemen A, van Dooren P, Huizinga BF, Hoofwijk AG. Randomized clinical trial comparing polypropylene or polydioxanone for midline abdominal wall closure. Br J Surg 2011;98:633-639.

2) van 't Riet M, Steyerberg EW, Nellensteyn J, Bonjer HJ, Jeekel J. Meta-analysis of techniques for closure of midline abdominal incisions. Br J Surg 2002;89:1350-1356.

3) Cobb WS, Harris JB, Lokey JS, McGill ES, Klove KL. Incisional herniorrhaphy with intraperitoneal composite mesh: a report of 95 cases. Am Surg 2003;69:784-787.

4) Anthony T, Bergen PC, Kim LT, et al. Factors affecting recurrence following incisional herniorrhaphy. World J Surg 2000;24:95-100; discussion 101.

5) Pollock AV, Evans M. Early prediction of late incisional hernias. Br J Surg 1989;76:953-954.

6) van't RM, De Vos Van Steenwijk PJ, Bonjer HJ, Steyerberg EW, Jeekel J. Incisional hernia after repair of wound dehiscence: incidence and risk factors. Am Surg 2004;70:281-286.

7) Luijendijk RW, Hop WC, van den Tol MP, et al. A comparison of 
suture repair with mesh repair for incisional hernia. N Engl J Med 2000;343:392-398.

8) Rosch R, Binnebosel M, Junge K, et al. Analysis of c-myc, PAI-1 and UPAR in patients with incisional hernias. Hernia 2008;12:285288.

9) Kingsnorth A, LeBlanc K. Hernias: inguinal and incisional. Lancet 2003;362:1561-1571.

10) George CD, Ellis H. The results of incisional hernia repair: a twelve year review. Ann R Coll Surg Engl 1986;68:185-187.

11) Forbes SS, Eskicioglu C, McLeod RS, Okrainec A. Meta-analysis of randomized controlled trials comparing open and laparoscopic ventral and incisional hernia repair with mesh. Br J Surg 2009;96:851-858.

12) Lomanto D, Iyer SG, Shabbir A, Cheah WK. Laparoscopic versus open ventral hernia mesh repair: a prospective study. Surg Endosc 2006;20:1030-1035.

13) Langer $S$, Christiansen J. Long-term results after incisional hernia repair. Acta Chir Scand 1985;151:217-219.

14) Ladurner R, Drosse I, Chiapponi $C$, et al. Polypropylene meshes coated with a polysaccharide based bioadhesive for intra-abdominal mesh fixation in a rabbit model. Surg Endosc 2013;27:19911996.

15) Cassar K, Munro A. Surgical treatment of incisional hernia. Br J Surg 2002;89:534-545.

16) Tuveri M, Tuveri A, Nicolo E. Repair of large abdominal incisional hernia by reconstructing the midline and use of an onlay of biological material. Am J Surg 2011;202:e7-11.

17) Millikan KW, Baptista M, Amin B, Deziel DJ, Doolas A. Intraperitoneal underlay ventral hernia repair utilizing bilayer expanded polytetrafluoroethylene and polypropylene mesh. Am Surg 2003;69:287-291; discussion 291-282.

18) Temudom $T$, Siadati M, Sarr MG. Repair of complex giant or recurrent ventral hernias by using tension-free intraparietal prosthetic mesh (Stoppa technique): lessons learned from our initial experience (fifty patients). Surgery 1996;120:738-743; discussion 743-734.

19) Ramirez OM, Ruas E, Dellon AL. "Components separation” method for closure of abdominal-wall defects: an anatomic and clinical study. Plast Reconstr Surg 1990;86:519-526.

20) Birolini C, Utiyama EM, Rodrigues AJ, Jr., Birolini D. Elective colonic operation and prosthetic repair of incisional hernia: does contamination contraindicate abdominal wall prosthesis use? J Am Coll Surg 2000;191:366-372.

21) LeBlanc KA, Booth WV, Whitaker JM, Bellanger DE. Laparoscopic incisional and ventral herniorrhaphy in 100 patients. Am J Surg 2000;180:193-197.

22) Shestak KC, Edington HJ, Johnson RR. The separation of anatomic components technique for the reconstruction of massive midline abdominal wall defects: anatomy, surgical technique, applications, and limitations revisited. Plast Reconstr Surg
2000;105:731-738; quiz 739.

23) Kingsnorth AN, Sivarajasingham N, Wong S, Butler M. Open mesh repair of incisional hernias with significant loss of domain. Ann R Coll Surg Engl 2004;86:363-366.

24) Tran NV, Petty PM, Bite U, Clay RP, Johnson CH, Arnold PG. Tissue expansion-assisted closure of massive ventral hernias. J Am Coll Surg 2003;196:484-488.

25) Rives J, Pire JC, Flament JB, Palot JP, Body C. [Treatment of large eventrations. New therapeutic indications apropos of 322 cases]. Chirurgie 1985;111:215-225.

26) Stoppa RE. The treatment of complicated groin and incisional hernias. World J Surg 1989;13:545-554.

27) Wantz GE. Incisional hernioplasty with Mersilene. Surg Gynecol Obstet 1991;172:129-137.

28) Ozturk G, Malya FU, Ersavas C, et al. A novel reconstruction method for giant incisional hernia: Hybrid laparoscopic technique. J Minim Access Surg 2015;11:267-270.

29) Dumanian GA, Denham W. Comparison of repair techniques for major incisional hernias. Am J Surg 2003;185:61-65.

30) Franklin ME, Dorman JP, Glass JL, Balli JE, Gonzalez JJ. Laparoscopic ventral and incisional hernia repair. Surg Laparosc Endosc 1998;8:294-299.

31) Korenkov M, Beckers A, Koebke J, Lefering R, Tiling T, Troidl H. Biomechanical and morphological types of the linea alba and its possible role in the pathogenesis of midline incisional hernia. Eur J Surg 2001;167:909-914.

32) Rosen M, Brody F, Ponsky J, et al. Recurrence after laparoscopic ventral hernia repair. Surg Endosc 2003;17:123-128.

33) Nguyen DH, Nguyen MT, Askenasy EP, Kao LS, Liang MK. Primary fascial closure with laparoscopic ventral hernia repair: systematic review. World J Surg 2014;38:3097-3104.

34) Kingsnorth AN. Laparoscopic incisional hernia repair with a self-centring suture (Br J Surg 2006; 93; 1549-1553). Br J Surg 2007;94:251.

35) van der Voort M, Heijnsdijk EA, Gouma DJ. Bowel injury as a complication of laparoscopy. Br J Surg 2004;91:1253-1258.

36) Schwartz MJ, Faiena I, Cinman N, et al. Laparoscopic bowel injury in retroperitoneal surgery: current incidence and outcomes. J Urol 2010;184:589-594.

37) Moreno-Egea A, Castillo JA, Girela E, Canteras M, Aguayo JL. Outpatient laparoscopic incisional/ventral hernioplasty: our experience in 55 cases. Surg Laparosc Endosc Percutan Tech 2002;12:171-174.

38) Heniford BT, Park A, Ramshaw BJ, Voeller G. Laparoscopic repair of ventral hernias: nine years' experience with 850 consecutive hernias. Ann Surg 2003;238:391-399; discussion 399-400.

39) Misiakos EP, Machairas A, Patapis P, Liakakos T. Laparoscopic ventral hernia repair: pros and cons compared with open hernia repair. Jsls 2008;12:117-125.

40) Deeken CR, Matthews BD. Ventralight ST and SorbaFix versus 
Physiomesh and Securestrap in a porcine model. Jsls 2013;17:549559 .

41) Cuccurullo D, Piccoli M, Agresta F, et al. Laparoscopic ventral incisional hernia repair: evidence-based guidelines of the first Italian Consensus Conference. Hernia 2013;17:557-566.

42) Kurmann A, Visth E, Candinas D, Beldi G. Long-term follow-up of open and laparoscopic repair of large incisional hernias. World J Surg 2011;35:297-301.

43) Orenstein SB, Dumeer JL, Monteagudo J, Poi MJ, Novitsky YW.
Outcomes of laparoscopic ventral hernia repair with routine defect closure using "shoelacing" technique. Surg Endosc 2011;25:14521457.

44) Bower CE, Reade CC, Kirby LW, Roth JS. Complications of laparoscopic incisional-ventral hernia repair: the experience of a single institution. Surg Endosc 2004;18:672-675.

45) Frezza EE, Shebani KO, Robertson J, Wachtel MS. Morbid obesity causes chronic increase of intraabdominal pressure. Dig Dis Sci 2007;52:1038-1041. 